
de camomila e densidade de semeadura na produção e composição do óleo essencial. Horticultura Brasileira 30: $195-200$.

\title{
Avaliação de germoplasma de camomila e densidade de semeadura na produção e composição do óleo essencial
}

\author{
Wanderlei do Amaral²; Cícero Deschamps'; Marília P Machado'; Henrique S Koehler'; Agnes de P \\ Scheer $^{3}$; Lílian Cocco ${ }^{3}$; Carlos Yamamoto ${ }^{3}$ \\ ${ }^{1}$ UFPR-Dep ${ }^{\text {to }}$ Fitotecnia e Fitossanitarismo, C. Postal 19061, 81531-990 Curitiba-PR; cicero@ufpr.br; ${ }^{2}$ Faculdades Integradas Espírita, \\ Curitiba-PR; ${ }^{3}$ UFPR-LACAUT-Dep ${ }^{\text {to }}$ Engenharia Química
}

\section{RESUMO}

O cultivo de camomila na região Sul do Brasil tem sido realizado utilizando-se como material genético a cultivar Mandirituba. Apesar da produção de capítulos florais e teor de óleos essenciais apresentado por esta cultivar ser considerado satisfatório, tem sido observada nas regiões produtoras a ocorrência de problemas fitossanitários que resultam em perdas significativas da produção. Este trabalho teve como objetivos comparar materiais genéticos de camomila provenientes da Holanda, Dinamarca e Mandirituba, e avaliar o efeito de diferentes densidades de semeadura no desenvolvimento das plantas, produção de capítulos, rendimento e qualidade do óleo essencial da cultivar Mandirituba, atualmente cultivada na região. Os experimentos foram conduzidos no município de Piraquara (PR). A extração do óleo essencial foi realizada por hidrodestilação em aparelho tipo Clevenger e a determinação da composição do óleo foi feita por cromatografia em fase gasosa acoplada ao espectrômetro de massa (GC/MS). Os materiais genéticos provenientes da Holanda e de Mandirituba apresentaram maior produção de biomassa seca de capítulos, em relação ao material genético da Dinamarca. A cultivar Mandirituba apresentou o maior rendimento de óleo essencial (4,71 $\left.\mu \mathrm{L} \mathrm{g}^{-1} \mathrm{~ms}\right)$, sendo também superior em relação à produtividade de óleo essencial $\left(2,40 \mathrm{~L} \mathrm{ha}^{-1}\right)$. Os materiais procedentes da Dinamarca e de Mandirituba apresentaram composição do óleo essencial semelhantes. A densidade de plantio de $1,0 \mathrm{~kg} \mathrm{ha}^{-1}$ da cultivar Mandirituba resultou em melhor desenvolvimento das plantas e maior produção de óleo essencial. Quantidades superiores a 3,0 $\mathrm{kg} \mathrm{ha}^{-1}$ de sementes não resultaram em maior densidade de plantas tendo em vista a alta competição e percentual de plântulas mortas.

Palavras-chave: Chamomilla recutita, espaçamento, óleo essencial, camazuleno, $\alpha$-bisabolol.

\begin{abstract}
Evaluation of germplasm and seeding rate over the production and composition of chamomile essential oil

The cultivar Mandirituba is the only genetic material for chamomile cultivation in southern Brazil. Besides the limitations regarding floral buds and the yield of essential oil presented by this cultivar, phytosanitary problems have also been observed. The objectives of this work were to compare the genetic materials from the Netherlands, Denmark and Mandirituba and to evaluate the effect of seeding rate on floral heads development and essential oil production, yield and quality. The experiments were carried out at field conditions in Piraquara, Paraná state, Brazil. The essential oil extraction was obtained by hydrodistillation using a Clevenger apparatus and the oil composition was analyzed by gas chromatography coupled to mass spectrometry (GC/MS). The genetic materials from the Netherlands and Mandirituba presented statistically higher dry mass accumulation than the ones from Denmark. However the material from Mandirituba showed a great essential oil yield $\left(4.7 \mu \mathrm{L} \mathrm{g}^{-1} \mathrm{~ms}\right)$ and productivity $\left(2.40 \mathrm{~L} \mathrm{ha}^{-1}\right)$. The genetic material from Denmark and Mandirituba showed similar essential oil composition. The density of $1.0 \mathrm{~kg}$ $\mathrm{ha}^{-1}$ for cultivar Mandirituba resulted on great plant development and essential oil production. The use of $3.0 \mathrm{~kg} \mathrm{ha}^{-1}$ of seeds did not increase plant density due to the high plant competition.
\end{abstract}

Keywords: Chamomilla recutita, density, essential oil, camazuleno, $\alpha$-bisabolol.

(Recebido para publicação em 1 de abril de 2011; aceito em 30 de março de 2012)

(Received on April 1, 2011; accepted on March 30, 2012)

\begin{abstract}
$\mathrm{A}$ camomila (Chamomilla recutita) é uma planta medicinal e aromática pertencente à família Asteraceae, com grande importância para a indústria devido à aplicação do óleo essencial na fabricação de aromatizante, sabonetes, perfumes, xampus e loções, e por conferir odor e sabor agradáveis a uma grande variedade de alimentos e bebidas. Possui também propriedades fitoterápicas im-
\end{abstract}

portantes tais como antiespasmódica, antiflogística, sedativa, antialérgica, vermífuga e emenagoga (Amaral et al., 2003; Mapeli et al., 2005).

Os óleos essenciais da camomila estão presentes nos capítulos florais e possuem propriedades aromáticas e medicinais, sendo identificados cerca de 120 constituintes químicos, compostos por 28 terpenóides, 36 flavonóides e 52 outras substâncias orgânicas (Salamón, 1992; Rodríguez et al., 1996; Teske \& Trentini, 1997; Silva Júnior, 2003).

No Paraná a produção de camomila está concentrada nos municípios de Mandirituba e São José dos Pinhais (Costa \& Doni-Filho, 2002). O seu cultivo nestas regiões é realizado utilizando-se somente a cultivar Mandirituba, que apresenta cerca de $0,86 \%$ de óleo 
essencial presente nos capítulos florais com 27,17 a $35,02 \%$ de camazuleno e de 7,13 a 11,72\% de $\alpha$-bisabolol (Corrêa Júnior, 1995).

A composição do óleo essencial de uma espécie aromática é determinada geneticamente, sendo geralmente específica para um determinado órgão e característica conforme seu estágio de desenvolvimento. Porém, condições ambientais podem induzir as variações significativas no rendimento e na qualidade do óleo essencial (Simões \& Spitzer, 1999). Dentre os fatores que influenciam a produção de óleo essencial, a competição das plantas por água, luz e nutrientes é diretamente definida pela densidade de semeadura. A existência de correlação entre a produção de óleos essenciais e fotossíntese e, portanto, com a produção de biomassa, foi verificada em Anethum graveolem, onde o aumento no nível de radiação elevou a produtividade e a produção de óleo essencial (Hälvä et al., 1992). Diversas recomendações de densidade de semeadura são encontradas para a camomila (Salamón, 1992; Silva Júnior, 2003; Ramos et al., 2004), porém poucos estudos foram realizados sobre o efeito da competição entre plantas na produção.

A avaliação de novos materiais genéticos é de extrema importância para melhorar o desempenho da cultura nas regiões produtoras tanto na produção de biomassa verde e capítulos florais como de óleos essenciais. Outros estudos como a competição de plantas permitem também determinar técnicas de manejo adequadas. Em se tratando da produção orgânica de camomila, a semeadura em linha, em densidade adequada, é de especial interesse para a produção orgânica de camomila, pois permite o controle mecânico de plantas daninhas, o que não é possível no sistema de semeadura a lanço o qual é dependente do controle químico.

Assim, os objetivos deste trabalho foram comparar os materiais genéticos e a composição do óleo essencial de camomila provenientes da Holanda, da Dinamarca e de Mandirituba, e avaliar o efeito de diferentes densidades de semeadura em linha no desenvolvimento, produção de capítulos teor e composição química do óleo essencial da cultivar
Mandirituba.

\section{MATERIAL E MÉTODOS}

Os experimentos foram realizados no Município de Piraquara (PR) (2532'07''S; 4903'54'W; altitude de $950 \mathrm{~m}$ ). O clima da região é classificado pelo sistema internacional de Köppen como subtropical mesotérmico úmido. $\mathrm{O}$ índice pluviométrico médio mensal foi de 98,8 milímetros, com temperaturas médias de $15^{\circ} \mathrm{C}$, onde a mínima registrada foi de $-1^{\circ} \mathrm{C}$ no mês de junho e a máxima de $31^{\circ} \mathrm{C}$ no mês de setembro (SIMEPAR, 2004). O solo da região é caracterizado como um Cambissolo hálico (EMBRAPA, 1999).

O solo em análise prévia possuía: $\mathrm{pH}$ $\mathrm{SMP}=5,05 ; \mathrm{Al}^{+3}=0,15 \mathrm{cmol}_{\mathrm{c}} \mathrm{dm}^{-3} ; \mathrm{H}^{+}$ $+\mathrm{Al}^{+3}=5,6 \mathrm{cmol}_{\mathrm{c}} \mathrm{dm}^{-3} ; \mathrm{K}^{+}=0,13 \mathrm{cmol}_{\mathrm{c}}$ $\mathrm{dm}^{-3} ; \mathrm{P}=3,40 \mathrm{mg} \mathrm{dm}^{-3} ; \mathrm{C}_{\text {orgânico }}=28,40$ $\mathrm{g} \mathrm{dm}^{-3}$; saturação por bases $=46,86 \%$.

A correção do solo foi realizada segundo as recomendações de Raij et al. (1996). Para a correção do pH, aplicou-se $2.500 \mathrm{~kg} \mathrm{ha}^{-1}$ de calcário tipo filler (100\% PRNT) a lanço, com posterior incorporação na profundidade de $0-10 \mathrm{~cm}$. Utilizou-se 40, 120 e $80 \mathrm{~kg}$ $\mathrm{ha}^{-1}$ de $\mathrm{N}, \mathrm{P}_{2} \mathrm{O}_{5}$ e $\mathrm{K}_{2} \mathrm{O}$, respectivamente, na forma de uréia, superfosfato triplo e cloreto de potássio.

Procedência e beneficiamento das sementes - Avaliou-se os materiais genéticos provenientes da Dinamarca, da Holanda e de Mandirituba. As sementes provenientes da Dinamarca foram cedidas pela empresa de sementes Vidasul, de Xanxerê (SC), lote $n^{\circ} 145385$, as da Holanda pela empresa Isla Sementes Ltda, de Porto Alegre (RS), lote $\mathrm{n}^{\mathrm{o}}$ 15860-HZ, e as sementes de camomila cv. Mandirituba foram fornecidas por um produtor rural do Município de Piraquara. As sementes da cv. Mandirituba foram beneficiadas, para a separação de impurezas e sementes chochas, em mesa de gravidade DAMAS ${ }^{\circledR}$ tipo Lanta-k, no Laboratório de Sementes da UFPR, em Curitiba (PR).

Análise dos materiais genéticos - Para a determinação da quantidade de sementes a serem plantadas por procedência, foram realizados testes de germinação em laboratório. Os testes foram conduzidos com 300 sementes (três lotes de 100 sementes) de cada procedência, distribuídas em caixas gerbox sobre duas folhas de papel mata-borrão e colocadas para germinar em câmara de germinação com temperatura de $20^{\circ} \mathrm{C}$. As avaliações foram feitas após 12 dias. Os materiais genéticos procedentes da Dinamarca e da Holanda apresentaram índices de germinação de $69 \%$ e $67 \%$, respectivamente, e o material procedente de Mandirituba apresentou índice de germinação de $35 \%$. A partir desses resultados foi realizado o plantio em linha com as quantidades de 2,8; 2,9 e 5,7 kg $\mathrm{ha}^{-1}$ de sementes viáveis para o material genético procedente da Dinamarca, Holanda e Mandirituba, respectivamente, de acordo com o índice de germinação de cada procedência, misturadas em 100 $\mathrm{kg} \mathrm{ha}^{-1}$ de fubá de milho como veículo utilizando-se espaçamento entre linhas de $0,50 \mathrm{~m}$.

O delineamento experimental utilizado foi em blocos casualizados, com três tratamentos e seis repetições, totalizando 18 parcelas, com 2 × $3 \mathrm{~m}$ cada, sendo 2,0 $\mathrm{m}^{2}$ de parcela útil, com distância de 1,0 m entre cada parcela.

Com relação ao desenvolvimento das plantas foram avaliados a biomassa seca dos capítulos, teor, produtividade ( $\mathrm{L} \mathrm{ha}^{-1}$ ) e composição do óleo essencial.

Para determinação da biomassa seca de capítulos utilizou-se estufa de secagem a $45^{\circ} \mathrm{C}$ por 24 horas. O teor de óleo essencial ( $\mu \mathrm{L} \mathrm{g}^{-1} \mathrm{~ms}$ ) foi determinado com a utilização de micropipeta.

Densidade de sementes - Os tratamentos consistiram de 1,$0 ; 2,0 ; 3,0$ e 4,0 $\mathrm{kg} \mathrm{ha}^{-1}$ de sementes beneficiadas da cultivar Mandirituba, sendo que $1,0 \mathrm{~kg} \mathrm{ha}^{-1}$ de sementes corresponde a 4.375.000 viáveis $\mathrm{ha}^{-1}$. A contagem das amostras de sementes foi realizada com auxílio de um microscópio estereoscópico, com posterior pesagem em balança de precisão, determinando assim o número de sementes por grama.

O espaçamento de semeadura adotado foi $0,50 \mathrm{~m}$ entre plantas, utilizando-se fubá de milho, na proporção de 50:1 como veículo para dispersar as sementes e o delineamento experimental foi inteiramente casualizado, com quatro tratamentos e cinco repetições em parcelas de 2 × $3 \mathrm{~m}$, com parcela útil de $2 \mathrm{~m}^{2}$. 
Durante o ciclo da cultura realizou-se apenas uma capina aos 30 dias após a emergência para controle de plantas invasoras.

Foram avaliados a altura média das plantas, número de hastes por planta, número de ramos na haste principal, biomassa fresca e seca das hastes, biomassa seca dos capítulos, diâmetro dos capítulos, altura dos capítulos, números de capítulos abertos e fechados, e número de plantas por metro linear.

Extração do óleo essencial- A extração do óleo essencial foi realizada por hidrodestilação durante 4 horas e 30 minutos em aparelho graduado tipo Clevenger (Wasicky, 1963), utilizando-se 46 g de capítulos secos. Após a extração, as amostras foram coletadas e armazenadas a $-20^{\circ} \mathrm{C}$ onde permaneceram até o momento das análises.

Para a quantificação dos constituintes, utilizou-se cromatógrafo gasoso Varian, modelo CP-3800, com detector FID (CG/FID). Para a separação cromatográfica, utilizou-se coluna capilar Chrompack, de sílica fundida CP-SIL PONA CB, 0,25 mm de diâmetro interno, $100 \mathrm{~m}$ de comprimento e $0,50 \mu \mathrm{m}$ de filme líquido. As condições de análise otimizadas para esta coluna foram: a) temperatura do injetor: $200^{\circ} \mathrm{C}$, split 1 : 100 ; b) quantidade de amostra injetada: 1,0 $\mu \mathrm{L}$; c) gás de arraste: hélio a 1,0 mL/ min. na temperatura de $120^{\circ} \mathrm{C}$; d) gás de make up: ar sintético, nitrogênio e hidrogênio; e) temperatura do detector FID: $300^{\circ} \mathrm{C}$; f) programação de temperatura do forno: $22 \mathrm{~min}$ a $120^{\circ} \mathrm{C}$, elevação de temperatura a $230^{\circ} \mathrm{C}$ na razão de $10^{\circ} \mathrm{C}$ permanecendo por $20 \mathrm{~min}$; g) tempo total de corrida: $53 \mathrm{~min}$. A identificação dos constituintes químicos foi realizada comparando-se a biblioteca da Nist98 (Varian Inc.) com os espectros de massa obtidos para cada composto (Borsato et al., 2005).

Análise estatística - As análises estatísticas foram feitas utilizando-se o programa MSTAT-C (Nissen, 1993). Os resultados foram submetidos à análise de variância e as médias testadas quanto à homogeneidade pelo teste de Bartlet e comparadas pelo teste de Tukey a 5\% de probabilidade.

\section{RESULTADOS E DISCUSSÃO}

Análise dos materiais genéticos - A biomassa seca dos capítulos do material genético proveniente da Dinamarca $\left(419,4 \mathrm{~kg} \mathrm{ha}^{-1}\right)$ foi significativamente inferior aos materiais provenientes da Holanda e Mandirituba (549,5 kg $\mathrm{ha}^{-1}$ e 510,0 $\mathrm{kg} \mathrm{ha}^{-1}$, respectivamente) (Tabela 1).

A cultivar Mandirituba apresentou teor de óleo essencial superior ao material genético da Dinamarca, porém sem diferença significativa em relação ao material genético da Holanda (Tabela 1). O teor de óleo essencial encontrado, para a cultivar de camomila Mandirituba, é considerado superior ao exigido na Farmacopéia Brasileira para a comercialização $(0,40 \%)$ (Wagner \& Bladt, 1995). Contudo, é mais baixo do que os teores da média mundial de $0,70 \%$, e também ao encontrado por Corrêa Júnior (1995) (0,80\%). Similarmente ao resultado encontrado para a cultivar Mandirituba em nosso estudo, Lopes et al. (2010) obtiveram aproximadamente $0,42 \%$ de óleo essencial de camomila em cultivo convencional; porém, no cultivo orgânico o teor de óleo essencial foi de $0,72 \%$, demonstrando que o ambiente, além do genótipo, influencia fortemente no teor de óleo essencial produzido pelas plantas. A produtividade de óleo essencial foi superior na cultivar Mandirituba, cuja média foi de 2,40 $\mathrm{L} \mathrm{ha}^{-1}$ (Tabela 1). Este fato pode estar relacionado com sua melhor adaptação a fatores edafoclimáticos, uma vez que a cultivar Mandirituba vem sendo cultivada na região por mais de três décadas. Trabalhos realizados com outras espé- cies também apresentaram diferenças no teor e rendimento de óleo essencial entre genótipos, como observado em Ocimum basilicum e Hyptis pectinata (Blank et al., 2004; Arrigoni-Blank et al., 2005).

Composição do óleo essencial - O material genético das três procedências não mostrou diferenças marcantes na sua composição. No entanto, houve variação na quantidade dos constituintes nos três materiais genéticos estudados.

O material proveniente da Dinamarca e a cultivar Mandirituba apresentaram maior porcentagem de $\alpha$-bisabolol $(12,77 \%$ e $11,31 \%$, respectivamente), sendo que o material da Holanda apresentou a maior porcentagem de cariofileno $(16,96 \%)$, de óxido de bisabolol A $(47,71 \%)$ e de oxido de bisabolona (6,17\%). O óleo essencial da cultivar Mandirituba apresentou maior porcentagem de óxido de bisabolol B (26,90\%), de camazuleno $(4,45 \%)$, de oxido de cariofileno $(3,26 \%)$, de 3 -careno $(0,60 \%)$ e de $\alpha$-pineno (2,93\%) em relação ao material genético da Holanda (Tabela 2). Apesar do resultado superior da cultivar Mandirituba a porcentagem de camazuleno encontra-se abaixo do esperado, pois a concentração média deste constituinte é de $10 \%$ (Borsato et al., 2008). As cultivares de camomila 'Soroksári 40', 'Lutea', 'Global' e 'Bona' produziram de 20,1\% a 24,4\% de camazuleno na Hungria (Gosztola et al., 2006).

A partir dos resultados, pode-se classificar o material genético procedente da Holanda como pertencente ao quimiotipo denominado camomila A ou tipo europeu, onde predomina o constituinte óxido de bisabolol A (Ta-

Tabela 1. Biomassa seca de capítulos, teor e produtividade de óleo essencial de materiais genéticos de camomila) (dry mass of flower buds, content and yield of essential oil of chamomile genetic material). Piraquara, UFPR, 2004.

\begin{tabular}{|c|c|c|c|}
\hline Procedência & $\begin{array}{l}\text { Biomassa seca dos } \\
\text { capítulos }(\mathrm{kg} / \mathrm{ha})^{1}\end{array}$ & $\begin{array}{l}\text { Teor de óleo essencial } \\
\qquad(\mu \mathrm{L} / \mathrm{g} \mathrm{ms})^{1}\end{array}$ & $\begin{array}{l}\text { Produtividade } \\
\text { de óleo }(\mathrm{L} / \mathrm{ha})^{1}\end{array}$ \\
\hline Dinamarca & $419,40 \mathrm{~b}$ & $2,62 \mathrm{~b}$ & $1,15 \mathrm{c}$ \\
\hline Holanda & $549,50 \mathrm{a}$ & $3,38 \mathrm{ab}$ & $1,83 \mathrm{~b}$ \\
\hline Mandirituba & $510,00 \mathrm{a}$ & $4,71 \mathrm{a}$ & $2,40 \mathrm{a}$ \\
\hline$\overline{\mathrm{CV}(\%)}$ & 34,45 & 19,04 & 38,43 \\
\hline
\end{tabular}

${ }^{1}$ Médias com a mesma letra nas colunas não diferem estatisticamente pelo teste de Tukey ao nível de $5 \%$ de probabilidade (means followed by the same letter do not differ by Tukey test at $5 \%$ ). 
Tabela 2. Composição de óleo essencial (\%) de camomila de diferentes materiais genéticos (composition of essential oil (\%) of chamomile genetic materials). Piraquara, UFPR, 2004.

\begin{tabular}{lrrrr}
\hline \multirow{2}{*}{ Constituintes } & \multicolumn{3}{c}{ Procedência } & \multirow{2}{*}{ CV(\%) } \\
\cline { 2 - 4 } & Dinamarca & Holanda & Mandirituba & \\
\hline a-pineno & $0,29 \mathrm{~B}$ & $0,00 \mathrm{C}$ & $2,93 \mathrm{~A}$ & 5,65 \\
3-careno & $0,00 \mathrm{~B}$ & $0,00 \mathrm{~B}$ & $0,60 \mathrm{~A}$ & 9,47 \\
Cariofileno & $15,43 \mathrm{~B}$ & $16,96 \mathrm{~A}$ & $9,26 \mathrm{C}$ & 10,55 \\
Oxido de Cariofileno & $2,93 \mathrm{~B}$ & $0,82 \mathrm{C}$ & $3,26 \mathrm{~A}$ & 9,03 \\
Óxido de bisabolol A & $19,14 \mathrm{~B}$ & $47,61 \mathrm{~A}$ & $16,83 \mathrm{C}$ & 12,98 \\
Óxido de bisabolol B & $26,84 \mathrm{~A}$ & $9,98 \mathrm{~B}$ & $26,90 \mathrm{~A}$ & 14,83 \\
$\alpha$-bisabolol & $12,77 \mathrm{~A}$ & $3,23 \mathrm{~B}$ & $11,31 \mathrm{~A}$ & 11,21 \\
Oxido de Bisabolona & $4,70 \mathrm{~B}$ & $6,17 \mathrm{~A}$ & $4,56 \mathrm{~B}$ & 10,46 \\
Camazuleno & $3,19 \mathrm{~B}$ & $2,83 \mathrm{~B}$ & $4,45 \mathrm{~A}$ & 5,90 \\
\hline
\end{tabular}

Valores seguidos pela mesma letra na linha não diferem estatisticamente entre si, pelo teste de Tukey ao nível de 5\% de probabilidade (values followed by same letter in the lines do not differ statistically by Tukey test, $\mathrm{p}<0.05)$.

bela 2), conforme Schilcher (1987). Os materiais procedentes da Dinamarca e de Mandirituba, por sua vez, podem ser considerados como pertencentes ao quimiotipo denominado camomila B ou tipo argentino, onde predomina oxido de bisabolol B (Tabela 2). O quimiotipo brasileiro é caracterizado por um teor de $0,6 \%$ de óleo essencial, contendo $1,91 \%$ de camazuleno, $16,20 \%$ de óxido de bisabolol A, $25,83 \%$ de óxido de bisabolol B e $16,05 \%$ de $\alpha$-bisabolol. Estes valores são semelhantes aos encontrados neste trabalho para o material genético procedentes da Dinamarca e da cultivar Mandirituba, cuja concentração de $\alpha$-bisabolol (11,31\%) foi semelhante aos valores descritos por Corrêa Júnior, (1995) para esta cultivar (7,13-11,72\%). No entanto, a porcentagem do constituinte camazuleno $(4,45 \%)$ foi inferior ao anteriormente descrito pelo mesmo autor $(27,17-35,02 \%)$. Para a camomila da competição, visto que ao utilizar 1,0 $\mathrm{kg} \mathrm{ha}^{-1}$ de sementes, a porcentagem de plântulas mortas foi de $24,8 \%$, enquanto que com $4,0 \mathrm{~kg} \mathrm{ha}^{-1}$ de sementes $63,5 \%$ das plântulas não sobreviveram.

A altura das plantas, número de hastes por planta e número de ramos na haste principal não foram influenciados pela densidade de semeadura (dados não mostrados), assim como a biomassa seca das plantas (Tabela 3). Dessa forma, pode-se inferir que o desenvolvimento vegetativo de camomila não foi afetado pelo aumento da densidade de semeadura.

A densidade de semeadura não teve efeito sobre o desenvolvimento dos capítulos florais. No entanto, a maior densidade resultou na formação de capítulos com menor diâmetro e maior altura (dados não mostrados). Além disso, observou-se uma redução do número de capítulos abertos com o aumento da densidade (Tabela 3), devido à tendência de diminuição do número de hastes por planta e número de ramos na haste principal em altas densidades de plantas.

Quanto à biomassa seca de capítulos, as médias foram semelhantes entre os tratamentos $\left(409,5-426,7 \mathrm{~kg} \mathrm{ha}^{-1}\right)$, a qual não foi influenciada pela densidade de semeadura (Tabela 3). Resultados divergentes foram obtidos com a mesma cultivar de camomila por Ramos et al. (2004), onde o menor espaçamento testado apresentou médias superiores de biomassa seca e número de capítulos florais, porém não interferiu na altura e diâmetro dos capítulos de camomila cv. 'Mandirituba'.

O teor e a produtividade de óleo essencial de camomila não tiveram influência na densidade de semeadura

Tabela 3. Desenvolvimento teor ( $\mu \mathrm{L} / \mathrm{g} \mathrm{ms})$ e produtividade ( $\mathrm{L} / \mathrm{ha})$ de óleo de camomila cv. Mandirituba em função de diferentes densidades de sementes (development, content ( $\mu \mathrm{L} / \mathrm{g} \mathrm{ms}$ ) and yield (L/ha) of chamomile oil depending on seeding rates). Piraquara, UFPR, 2004.

\begin{tabular}{|c|c|c|c|c|}
\hline $\begin{array}{l}\text { Densidade de } \\
\text { semeadura (kg/ha) }\end{array}$ & $\begin{array}{c}\text { Plantas } / \mathbf{m}^{2} \\
\left(\mathrm{n}^{0}\right)^{1}\end{array}$ & $\begin{array}{c}\text { Biomassa seca das plan- } \\
\text { tas }\left(\mathrm{kg} / \mathrm{m}^{2}\right)^{\mathrm{ns}}\end{array}$ & 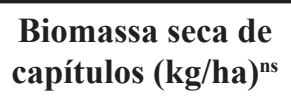 & $\begin{array}{l}\text { Capítulos abertos/ } \\
\text { planta }\left(\mathrm{n}^{0}\right)^{1}\end{array}$ \\
\hline 1,0 & $329,00 \mathrm{~b}$ & 0,199 & 422,3 & $5,65 \mathrm{ab}$ \\
\hline 2,0 & $533,33 a b$ & 0,268 & 411,0 & $6,44 \mathrm{a}$ \\
\hline 3,0 & $553,66 \mathrm{ab}$ & 0,217 & 409,5 & $5,22 \mathrm{~b}$ \\
\hline 4,0 & $640,32 \mathrm{a}$ & 0,218 & 426,7 & $4,47 \mathrm{c}$ \\
\hline CV (\%) & 24,72 & 32,25 & 23,71 & 18,79 \\
\hline
\end{tabular}

${ }^{1}$ Médias com a mesma letra minúscula nas colunas não diferem estatisticamente pelo teste de Tukey ao nível de 5\% de probabilidade (means

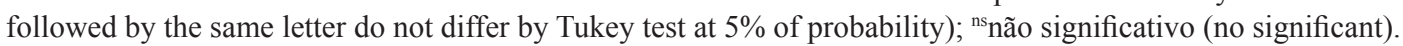


Tabela 4. Desenvolvimento, teor ( $\mu \mathrm{L} / \mathrm{g} \mathrm{ms})$ e produtividade (L/ha) de óleo de camomila cv. Mandirituba em função de diferentes densidades de sementes (development, content ( $\mu \mathrm{L} / \mathrm{g} \mathrm{ms}$ ) and yield (L/ha) of chamomile oil depending on seeding rates). Piraquara, UFPR, 2004.

\begin{tabular}{|c|c|c|c|}
\hline $\begin{array}{l}\text { Densidade de semeadura } \\
(\mathrm{kg} / \mathrm{ha})\end{array}$ & $\begin{array}{c}\text { Capítulos/planta }{ }^{1} \\
\left(\mathrm{n}^{0}\right)\end{array}$ & $\begin{array}{c}\text { Teor de óleo essencial } \\
(\mu \mathbf{L} / \mathbf{g} \mathbf{~ m s})^{\mathrm{ns}}\end{array}$ & $\begin{array}{c}\text { Produtividade de óleo } \\
\text { essencial (L/ha) }{ }^{\text {ns }}\end{array}$ \\
\hline 1,0 & $6,64 \mathrm{a}$ & 5,60 & 2,37 \\
\hline 2,0 & $7,34 \mathrm{a}$ & 5,33 & 2,21 \\
\hline 3,0 & $6,05 \mathrm{ab}$ & 5,06 & 2,03 \\
\hline 4,0 & $5,12 b$ & 4,86 & 2,05 \\
\hline CV (\%) & 11,65 & 15,67 & 28,37 \\
\hline
\end{tabular}

${ }^{1}$ Médias com a mesma letra minúscula nas colunas não diferem estatisticamente pelo teste de Tukey ao nível de $5 \%$ de probabilidade (means

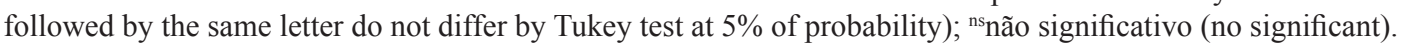

Tabela 5. Composição de óleo essencial (\%) de camomila, cv. Mandirituba em função de diferentes densidades de semeadura (essential oil composition (\%) of chamomile cultivar Mandirituba depending on seeding rates). Piraquara, UFPR, 2004.

\begin{tabular}{lrrrrr}
\hline \multirow{2}{*}{ Constituintes } & \multicolumn{3}{c}{ Densidade de semeadura (kg/ha) } & \multirow{2}{*}{$\mathbf{C V}(\mathbf{\%})$} \\
\cline { 2 - 4 } & \multicolumn{1}{c}{$\mathbf{1 , 0}$} & $\mathbf{2 , 0}$ & $\mathbf{3 , 0}$ & \multicolumn{1}{c}{$\mathbf{4 , 0}$} & \\
\hline a-pineno & $1,32 \mathrm{~A}$ & $1,55 \mathrm{~A}$ & $1,61 \mathrm{~A}$ & $0,96 \mathrm{~B}$ & 8,32 \\
Cariofileno & $19,71 \mathrm{~A}$ & $19,19 \mathrm{~A}$ & $17,67 \mathrm{~B}$ & $21,15 \mathrm{~A}$ & 3,11 \\
Oxido de Cariofileno & $3,58 \mathrm{~A}$ & $3,39 \mathrm{~A}$ & $2,94 \mathrm{C}$ & $3,21 \mathrm{~B}$ & 2,07 \\
Óxido de bisabolol A & $18,99 \mathrm{~A}$ & $19,49 \mathrm{~A}$ & $20,82 \mathrm{~A}$ & $17,38 \mathrm{~B}$ & 6,75 \\
Óxido de bisabolol B & $21,32 \mathrm{~A}$ & $22,23 \mathrm{~A}$ & $23,68 \mathrm{~A}$ & $23,77 \mathrm{~A}$ & 1,40 \\
$\alpha$-bisabolol & $10,38 \mathrm{~A}$ & $11,02 \mathrm{~A}$ & $10,53 \mathrm{~A}$ & $9,72 \mathrm{~B}$ & 2,33 \\
Oxido de Bisabolona & $3,97 \mathrm{~B}$ & $3,99 \mathrm{~B}$ & $5,22 \mathrm{~A}$ & $4,50 \mathrm{~B}$ & 8,75 \\
Camazuleno & $5,80 \mathrm{~A}$ & $5,05 \mathrm{~A}$ & $5,25 \mathrm{~A}$ & $5,49 \mathrm{~A}$ & 2,88 \\
\hline
\end{tabular}

Valores seguidos pela mesma letra na linha não diferem estatisticamente entre si, pelo teste de Tukey ao nível de 5\% de probabilidade (values followed by same letter in the lines do not differ statistically by Tukey test, $\mathrm{p}<0.05$ ).

(Tabela 4). Mas é interessante destacar que houve redução no rendimento e produção de óleo essencial quando a densidade de semeadura foi maior. Enquanto na menor densidade utilizada $\left(1,0 \mathrm{~kg} \mathrm{ha}^{-1}\right.$ de sementes) obteve-se 2,37 $\mathrm{L} \mathrm{ha}^{-1}$ de óleo essencial, na maior densidade $\left(4,0 \mathrm{~kg} \mathrm{ha}^{-1}\right.$ de sementes) obteve-se 2,05 L ha-1 (Tabela 4). Devido ao aumento da competição o número e o diâmetro dos capítulos foram menores ocorrendo, consequentemente, redução da produtividade de óleo essencial. Estes resultados indicam que a menor densidade de plantio em linha conforme avaliado é suficiente para a implantação da cultura a campo, considerando o desenvolvimento vegetativo e produção de óleo essencial que são características importantes se o destino da produção for o mercado de chás. Segundo Munarin et al. (2010), a pressão populacional diminui a capacidade produtiva total das plantas, devido à competição por fatores de crescimento, tais como radiação solar fotossinteticamente ativa, água e nutrientes, o que acarreta em decréscimo da produtividade. Independente da densidade de semeadura pode-se considerar que a produtividade de óleo essencial de camomila cv. Mandirituba foi baixa, uma vez que a mesma cultivar obteve uma produtividade de $6,56 \mathrm{~L} \mathrm{ha}^{-1}$ de óleo essencial no município de Campo Magro (PR), conforme Nalepa \& Carvalho (2007). No Sul da Índia registrou-se uma produção de $6,36 \mathrm{~L} \mathrm{ha}^{-1}$ de óleo essencial da camomila de procedência alemã, quando se utilizou espaçamento de 30x20 cm (Kanjilal \& Singh, 2000; Nidagundi \& Hegde, 2007).

A maioria dos constituintes do óleo essencial tiveram seus níveis reduzidos na maior densidade de semeadura, indicando que a qualidade deste pode ser afetada no plantio em altas densidades (Tabela 5). Os componentes camazuleno e $\alpha$-bisabolol que possuem maior importância econômica no óleo essencial de camomila apresentaram níveis su- periores nas densidades de 1,0 e $2,0 \mathrm{~kg}$ ha $^{-1}$ de sementes ha ${ }^{-1}$, respectivamente. De forma contrária, os precursores do $\alpha$-bisabolol (bisabolol óxidos A e B) foram superiores em altas densidades. Estes resultados diferem daqueles encontrados por Sváb et al. (1967) que não observaram diferenças significativas na porcentagem de camazuleno no óleo essencial em capítulos florais de cultivares de camomila da Hungria e do Egito, sob a influência de diferentes populações $\left(1,6 ; 3,0\right.$ e 5,3 plantas $\left.\mathrm{m}^{-2}\right)$.

Conclui-se que no município de Piraquara, sob as condições em que se realizou o presente estudo, a cultivar Mandirituba mostrou-se mais adequada para a produção de óleo essencial de camomila, devido à maior produtividade apresentada. No entanto, são necessários mais estudos com relação ao manejo da cultura para elevar a produtividade do óleo essencial de diferentes materiais genéticos, uma vez que o produtor encontra-se na dependência de uma 
única cultivar. Além disso, o material genético da Dinamarca obteve resultados semelhantes à cultivar Mandirituba em relação à maioria dos constituintes presentes no óleo essencial.

Considerando os resultados referentes ao desenvolvimento vegetativo e de capítulos florais, teor e qualidade de óleo essencial, e a questão econômica do custo das sementes, a densidade adequada para o plantio em linha diretamente a campo é de $1,0 \mathrm{~kg} \mathrm{ha}^{-1}$ para a cv. Mandirituba. O plantio em densidades superiores a $3 \mathrm{~kg} \mathrm{ha}^{-1}$ de sementes não resulta em maior densidade de plantas, tendo em vista a alta competição e percentual de plântulas mortas.

\section{REFERÊNCIAS}

AMARAL FMM; COUTINHO DF; RIBEIRO MNS; OLIVEIRA MA. 2003. Avaliação da qualidade de drogas vegetais comercializadas em São Luíz - Maranhão. Revista Brasileira de Farmacognosia 13: 27-30.

AMARAL W; DESCHAMPS C; FAVARETTO $\mathrm{N}$; KOELER HS; SHEER AP; YAMAMOTO C; CÔCCO CL. 2008. Desenvolvimento, rendimento e composição de óleo essencial de camomila [Chamomilla recutita (L.) Rauschert] sob adubação orgânica e mineral. Revista Brasileira de Plantas Medicinais 10: $1-8$.

ARRIGONI-BLANK MF; SILVA-MANN R; CAMPOS DA; SILVAPA; ANTONIOLLIAR; CAETANO LC; SANTANA AEG; BLANK AF. 2005. Morphological, agronomical and pharmacological characterization of Hyptis pectinata (L) poit germplasm. Revista Brasileira de Farmacognosia 15: 298-303.

BLANK AF; CARVALHO FILHO JLS; SANTOS NETO AL; ALVES PB; ARRIGONI-BLANK MF; SILVA-MANN R; MENDONÇA MC. 2004. Caracterização morfológica e agronômica de acessos de manjericão e alfavaca. Horticultura Brasileira 22: 113-116.

BORSATO AV; DONI-FILHO L; CÔCCO LC. 2005. Secagem da camomila [Chamomilla recutita (L.) Raeuchert] com cinco vazões específicas do ar. Revista Brasileira de Plantas Medicinais 7: 65-71.

BORSATO AV; DONI-FILHO L; MIGUEL
OG; PAGLIA EC. 2008. Propriedades físicoquímicas do óleo essencial de camomila [Chamomilla recutita (L.) Rauschert] submetida à secagem em camada fixa. Revista Brasileira de Plantas Medicinais 10: 24-30.

CORRÊA JÚNIOR C. 1995. "Mandirituba": nova cultivar brasileira de camomila. Horticultura Brasileira 13: 61.

COSTA MAD; DONI-FILHO L. 2002. Aspectos do processo de produção agrícola na cultura da camomila [Chamomilla recutita (L.) Rauschert] no Município de Mandirituba, Paraná. Visão Acadêmica 3: 49-56.

EMBRAPA. Centro Nacional de Pesquisa de Solos. 1999. Sistema Brasileiro de Classificação de Solos. Rio de Janeiro: Embrapa-CNPS. 412p.

GOSZTOLA B; NÉMETH É; SÁROSI S; SZABÓ K; KOZAK A. 2006. Comparative evaluation of chamomile (Matricaria recutita L.) populations from different origin. International Journal of Horticultural Science 12: 91-95.

HÄLVÄ S; CRAKER LE; SIMON JE; CHARLES DJ. 1992. Light levels, growth and essential oil in dill (Anethum graveolem L.). Journal of Herbs, Spices and Medicinal plants 1: 47-58.

KANJILAL PB; SINGH RS. 2000. Effect of spacing and planting time on chamomile performance. Indian Journal of Agricultural Sciences 70: 259-260.

LOPES AP; SANTOS RF; LUCCA PSR; ECKERT RG. 2010. Manejos orgânico e convencional da camomila visando à produção de óleo essencial. Revista Varia Scientia Agrária 1: 45-54.

MAPELI NC; VIEIRA MC; HEREDIA ZNA; SIQUEIRA JM. 2005. Produção de biomassa e de óleo essencial dos capítulos florais da camomila em função de nitrogênio e fósforo. Horticultura Brasileira 23: 42-46.

MUNARIN EEO; HEREDIA ZÁRATE NA; VIEIRA MC; ROSA YBCJ; RODRIGUES ET. 2010. Espaçamento entre plantas e cobertura do solo com cama-de-frango na produção de bardana (Arctium lappa L.). Revista Brasileira de Plantas Medicinais 12: 141-148.

NALEPA T; CARVALHO RIN. 2007. Produção de biomassa e rendimento de óleo essencial em camomila cultivada com diferentes doses de cama-de-aviário. Scientia Agraria 8: 161-167.

NIDAGUNDI R; HEGDE L. 2007. Cultivation prospects of German chamomile in South India. Natural Product Radiance 6: 135-137.

NISSEN O. 1993. MSTAT-C - A Microcomputer for design, management, and analysis of agronomic research experiments. Version 2.11. East Lansing, Michigan State University. 300p.
RAIJ BV; CANTARELLA H; GUAGGIO JA; FURLANI AMC. 1996. Recomendações de adubação e calagem para o Estado de São Paulo. 2.ed., Campinas: Instituto Agronômico \& Fundação IAC. 285p.

RAMOS MBM; VIEIRA MC; HEREDIA ZNA; SIQUEIRA JM; ZIMINIANI MG 2004. Produção de capítulos florais em função de populações de plantas e da incorporação ao solo de cama-de-aviário. Horticultura Brasileira 22: 566-572.

RODRÍGUEZFM; MOURELLEJF; GUTIÉRREZ ZP. 1996. Actividad espasmolitica del extrato fluido de Matricaria (Manzanilla) en organos ailados. Revista Cubana de Plantas Medicinales 1: 19-24.

SALAMÓN I. 1992. Production of chamomile [Chamomilla recutita (L.) Rauschert] in Czecho-Slovakia. Journal of Herbs, Spices and Medicinal Plants 1: 37-45.

SCHILCHER H. 1987. Die Kamille. Wissenschaftliche Verlagsgesellschaft, Stuttgart 152p.

SHAMS-ARDAKANI M; GHANNADI A; RAHIMZADEH A. 2006. Volatile constituents of Matricaria chamomilla L. from Isfahan, Iran. Iranian Journal of Pharmaceutical Sciences 2: 57-60.

SILVA JÚNIOR AA. 2003. Essentia Herba: Plantas Bioativas. Florianópolis: Epagri, 1v. 441p.

SIMEPAR - Sistema Meteorológico do Paraná, Estação Meteorológica de Pinhais, Boletim. Curitiba, 2004.

SIMÕES CMO; SPITZER V. Óleos voláteis. In: SIMÕES CMO. et al. 1999, Farmacognosia: da planta ao medicamento. 2.ed., Porto Alegre: UFPR, p. 387-416.

SVÁB J; EL-DIN-AWAAD C; FAHMY F. 1967. The influence of highly different ecological effects on the volatile oil content and composition in the chamomile. Herba Hungárica 6: 177-188.

TAKAHASHI LSA; ROCHA JN; SOUZA JRP. 2006. Revisão sobre produção e tecnologia de sementes de espécies medicinais. Revista Brasileira de Plantas Medicinais 8: 198-209.

TESKE M; TRENTINI AMM. 1997. Herbarium compêndio de Fitoterapia. Curitiba: Herbarium Laboratório Botânico 69 - 71 .

WAGNER JC; BLADT S. (1995). Plant drug analysis: a thin layer chromatography atlas. 2 ed., Berlin: Springer, p.384.

WASICKY R. 1963. Uma modificação do aparelho de clevenger para extração de óleos essenciais. Revista Faculdade de Farmácia e Bioquímica 1: 77-81. 